\title{
The Association Between Alkaline Phosphatase and Neurological Outcome in Patients with Post-cardiac Arrest: a Secondary Analysis of Data from Belgium
}

jiacheng he ( $\nabla$ garxin@tom.com )

People's Hospital of Nanhai District,Foshan City

Research Article

Keywords: alkaline phosphatase,neurological outcome,cardiac arrest

Posted Date: July 30th, 2021

DOI: https://doi.org/10.21203/rs.3.rs-744547/v1

License: (a) (i) This work is licensed under a Creative Commons Attribution 4.0 International License. Read Full License 


\section{Abstract}

Background: Prognostication of the unfavourable neurological outcome(UNO) after Cardiac arrest(CA) is multimodal while blood biomarkers are an attractive option.Serum alkaline phosphatase(ALP) is shown to be associated with ischemic stroke and considered as an independent prognostic factor for long-term functional outcome after acute cerebral infarction. We aimed to study the association between ALP and UNO in 3 months in patients after CA.

Methods:Review of consecutive patients admitted to the ICU at Erasme Hospital, Brussels (Belgium) following CA between January 2007 and December 2015. The outcome was the UNO in 3 months. We used multivariable logistic regression models to calculate the adjusted odd ratio (OR) with $95 \%$ confidence interval $(\mathrm{Cl})$. Interaction and stratified analyses were conducted according to Shock,Dobutamine.used,Hypertension,Cardiac.Etiology and Shockable.Rhythm.

Results: We included 374 CA patients. The multivariate logistic regression analyses revealed that the risk of UNO in 3 months after CA was significantly associated with ALP.When ALP was Equal is divided in 3 groups, the risk of ALP>91 level and adverse outcomes was 1.7407 times of ALP $<64$ level ( $P$ for trend=0.19709). In the non-hypertension group, the risk of adverse outcomes increased with increased ALP by 1.018 (1.0041.01,1.032).

Conclusions: ALP is an independent risk factor for the UNO in 3 months after CA, especially in non-hypertension. Elevated ALP was significantly associated with increased UNO in 3 months after CA. However, the prediction significance of ALP for long-term neurological outcome in patients after CA is needed further studied.

\section{Introduction}

Cardiac arrest (CA) which had an annual incidence of nearly 350,000 out-of-hospital and about 750,000 in-hospital events is common in the United States ${ }^{1,2}$.Even after the rescue, the ischemia-reperfusion injury that occurs after the return of spontaneous circulation (ROSC) contributes to a systemic inflammatory which further increases the likelihood of a poor outcome including hypoxic-ischemic brain injury.Neurological prognostication after CA is challenging due to the lack of early and reliable predictors for clinically relevant brain damage.The prediction of brain injury is multimodal, including repeated clinical neurological examinations, diagnostic imaging, neurophysiological tests, and blood biomarkers ${ }^{3}$.Among blood biomarkers are an attractive choice because they are not affected by sedative drugs and are considered objective measures.Currently, the only recommended biomarkers after cardiac arrest is neuron-specific enolase evaluated $48-72 \mathrm{~h}$ after $\mathrm{CA}^{4}$. But earlier, simpler and reliable outcome predictors are needed to stratification and improve care after $\mathrm{CA}^{5}$.

Alkaline phosphatase (ALP), an enzyme first discovered in the 1920 s, has been evaluated as a potential biomarker for many diseases ${ }^{6}$. There are four isoenzymes in humans. Intestinal tract (IAP), placenta, germinal tissue (GCAP), and tissue-specific (TNAP).TNAP can be expressed through a variety of tissues, including multiple cell types in the brain ${ }^{7}$.Ermonval et al and Brun-Heath et al have suggested that ALP could be expressed in cerebral endothelial cells and neurons $s^{8,9}$. Lee et al suggested that the increase of ALP at stroke is related to the degree of cerebral cerebrovascular disease ${ }^{10}$.Ischemic stroke is similar to neurological injury after CA.Brain cells are very sensitive to hypoxia.After Ischemic stroke, ischemic stoves will have ischemic and hypoxic injury, if reperfusion treatment can be achieved, and reperfusion injury.After CA occurs, blood supply to the whole brain stopped, and ischemia and hypoxia injury to the brain, when effective CRP or ROSC, the brain recovered blood supply, followed by reperfusion injury.Therefore, we argue that the brain after CA suffers blows of the same nature as ischemic stroke.Several studies have also noted that ALP is associated with ischemic stroke as an independent prognostic factor for the long-term functional outcome after acute cerebral infarction ${ }^{11-13}$.

Thus, the goal of this study is to study the relationship between ALP and post-CA neural results, and also to evaluate the effects of other classical risk factors on associations through interaction and hierarchical analysis.

\section{Methods}

\section{Data Source}

Date were obtained from 'DATADRYAD' database (www.Datadryad.org). This website permitted users to freely download the raw data. According to Dryad Terms of Service, we cited Dryad data package in the present study ${ }^{14}$."Data from: Acute liver dysfunction after cardiac arrest," Dryad, ataset, https://datadryad.org/stash/dataset/doi:10.5061/dryad.qv6fp83)in the present study. Authors of the original study have waived all copyright and related ownership of these data. Therefore, we could use these data for secondary analysis without infringing on the authors' rights.

\section{Study Design and Participants}

This retrospective study was performed in the Department of Intensive Care at Erasme Hospital, Brussels (Belgium). 435 comatose patients (Glasgow Coma Scale, GCS < 9) admitted after in-hospital CA (IHCA) or out-of-hospital CA(OHCA) were included in a prospective institutional database (January 2007 to December 2015) and considered as eligible for the study ${ }^{14}$.All methods were performed in accordance with the relevant guidelines and regulations, as lesu $E$, Franchi F, Zama CF, et al. described previously ${ }^{14}$. The data we got has been initially screened, as follows: excluded participants who were died less than 24 hours $(n=51)$ after ICU admission, and missing data on liver function $(n=10)^{14}$.The local Ethical Committee (Comite' d'Ethique HospitaloFacultaire ErasmeULB) approved the study (P2017/264) but waived the need for informed consent because of its retrospective nature.

\section{Post-resuscitation care}


The protocol of post-resuscitation management had been extensively described elsewhere ${ }^{15}$. Advanced hemodynamic monitoring was used (PiCCO, Pulsion, Munich, Germany) and cardiac function was assessed by repeated transesophageal and/or transthoracic echocardiography. Mean arterial pressure (MAP) was maintained at $>65-70 \mathrm{mmHg}$ using volume resuscitation, dobutamine, and/or noradrenaline, whenever needed. Intra-aortic balloon counterpulsation (IABP) or extracorporeal membrane oxygenation (ECMO) was also used in cases of severe cardiogenic shock. Ventilation was set to maintain normocapnia and $\mathrm{SpO}_{2}>94 \%$. Blood glucose was kept between 110 and $150 \mathrm{mg} / \mathrm{dl}$ using a continuous insulin infusion. Enteral nutrition was initiated during TTM and continued thereafter according to gastric tolerance. Neurological monitoring and prognostication of coma after CA were performed using a multimodal approach, as previously described ${ }^{16}$.

\section{Data Collection and Measurements}

We collected data on demographics (including chronic use of any anticoagulant), preexisting chronic diseases, cardiopulmonary resuscitation (CPR) (initial rhythm, bystander CPR, time-to-ROSC, total-adrenaline-dose), and blood test in all patients. Blood samples were analyzed for Glutamic-oxaloacetic transaminase (GOT, normal values $\leq 50 \mathrm{IU} / \mathrm{L})$, Glutamic-pyruvic transaminase (GPT, normal values $\leq 50 \mathrm{IU} / \mathrm{L})$, lactate dehydrogenase (LDH, normal values $<200 \mathrm{IU} / \mathrm{L}$ ), prothrombin time (PT, normal values $>70 \%$ ), international normalized ratio (INR, normal values $=<1.2$ ), total bilirubin (normal values $=<1.2$ $\mathrm{mg} / \mathrm{dl}$ ) and fibrinogen (normal values $>150 \mathrm{mg} / \mathrm{dl}$ ) on admission and then once daily until ICU discharge.

As described before ${ }^{14}$, Neurological evaluation was assessed at 3 months after CA using the cerebral performance categories score $(\mathrm{CPC} ; 1=$ no or mild neurological disability, 2 = moderate neurological disability, 3 = severe neurological impairment, 4 = vegetative state, $5=$ death) ${ }^{17}$. Unfavorable neurological outcome at 3 months after CA(Unfavourite Outcome in 3 months) was considered as a CPC 35 ; favorable neurological outcome as CPC $1-2^{18}$.The shock was defined as the need for vasopressor agents for more than 6 hours.

\section{Statistical Analysis.}

All normally distributed and skewed continuous variables were presented as mean (SD) or median (interquartile range [IQR]). Categorical variables were indicated as frequencies (\%). For baseline characteristics analysis, the statistical differences among tertile groups of ALP were tested with one-way ANOVA for continuous variables and chi-square test for categorical variables. The effect of ALP on unfavourable neurological outcome(UNO) in 3 months was evaluated using logistic regression models (odd ratio [OR] and 95\% confidence interval [CI]).Both nonadjusted and multivariate-adjusted models were used. To assess confounding, we entered covariates into a binary logistic regression model in the basic model or eliminated the covariates in the complete model one by one and compared the regression coefficients ${ }^{23}$.

Variables thought to be confounders based on existing literature and clinical judgment were included ${ }^{19-22}$.In this study, the logistic regression models were adjusted for age+gender+Weight+Witnessed.Arrest+Bystander.CPR+time.to.ROSC+

Epinephrine.total.dose+Out.of.Hospital+Cardiac.Etiology+Shockable.Rhythm+Corticoids+Chronic.Anticoagulation+Chron.Heart.Failure+Hypertension+Coron.Ar trends were conducted with linear regression by entering the median value of each ALP tertile as a continuous variable in the models.

Logistic regression model with cubic spline functions and the smooth curve fitting were used to identifying linear relationships between ALP and UNO in 3 months. The subgroup analyses were conducted by Dobutamine.used and Shock using stratified logistic regression

model. Interaction across subgroups was tested using the likelihood ratio test. For all statistical analyses, we used statistical packages $\mathrm{R}$ version 3.4 .3 (The $\mathrm{R}$ Foundation, Vienna, Austria) and EmpowerStats (X\&Y Solutions, Inc., Boston, MA, USA). P< 0.05 was considered statistically significant.

For other continuous variables with missing values less than $6 \%$, the missing values were replaced by the mean values.

\section{Results}

\section{Population}

435 comatose patients (Glasgow Coma Scale, GCS < 9) admitted after IHCA or OHCA were included.After excluding participants who were died less than 24 hours $(n=51)$ after ICU admission, and missing data on liver function $(n=10)$ because of ALP being a kind of liver enzyme, and we needed to adjust the indexes related to liver.The final cohort included 374 patients. The flow chart of the study patients selection is presented in Figure 1.

\section{Baseline Characteristics of Study Participants by Categories of Serum ALP Levels}

The baseline characteristics of all participants were listed in Table 1.The ALP is evenly divided into 3 groups based on the serum ALP.226 (60.4\%) patients had an UNO in 3 months.159(42.5\%) patients had hypertension.221(59.1\%) patients had cardiac etiology.153(40.9\%) patients had shockable rhythm during CA.200(53.5\%) participants suffered shock during ICU stayed,and 201 (53.7\%) participants used Dobutamine during ICU stayed. The mean ALP level was 93.7 \pm 66.7 IU/L. Participants with higher serum ALP levels were more likely to be associate with IHCA, Shockable.Rhythm, Corticoids, Chronic Renal Failure, Shock, Dobutamine.used,VSP, AKI, CRRT.used, higher CRP, higher Cr, higher GOT, higher GGT, and higher BIL 
Table 1

Baseline characteristics of individuals stratified by tertiles of baseline serum ALP level

\begin{tabular}{|c|c|c|c|c|c|}
\hline \multirow[t]{2}{*}{ Variables } & \multirow[t]{2}{*}{ Total $(n=374)$} & \multicolumn{4}{|c|}{ Serum ALP (U/L) } \\
\hline & & $<64(n=125)$ & $64-91(n=121)$ & $>91(n=128)$ & P Value \\
\hline Men (\%) & $104(27.8)$ & $40(32)$ & $24(19.8)$ & $40(31.2)$ & 0.058 \\
\hline Age (yr) & $61.8 \pm 15.4$ & $59.5 \pm 15.9$ & $63.8 \pm 15.3$ & $62.2 \pm 14.8$ & 0.09 \\
\hline Weight(kg) & $77.0 \pm 14.7$ & $76.0 \pm 15.3$ & $77.7 \pm 12.7$ & $77.4 \pm 15.9$ & 0.611 \\
\hline ICU.stay(day) & $7.9 \pm 9.7$ & $7.9 \pm 8.8$ & $7.1 \pm 7.7$ & $8.8 \pm 12.0$ & 0.408 \\
\hline Witnessed.Arrest(\%) & $320(85.6)$ & $110(88)$ & $99(81.8)$ & $111(86.7)$ & 0.348 \\
\hline Bystander.CPR(\%) & $254(67.9)$ & $88(70.4)$ & $77(63.6)$ & $89(69.5)$ & 0.467 \\
\hline time.to.ROSC(min) & $18.0 \pm 14.1$ & $18.9 \pm 16.3$ & $18.2 \pm 13.5$ & $17.1 \pm 12.3$ & 0.613 \\
\hline Epinephrine.total.dose(mg) & $4.1 \pm 3.7$ & $4.2 \pm 4.0$ & $4.0 \pm 3.8$ & $3.9 \pm 3.2$ & 0.841 \\
\hline Out.of.Hospital(\%) & $207(55.3)$ & $75(60)$ & $74(61.2)$ & $58(45.3)$ & 0.019 \\
\hline TTM(\%) & $331(88.5)$ & $116(92.8)$ & $106(87.6)$ & $109(85.2)$ & 0.152 \\
\hline Cardiac.Etiology & $221(59.1)$ & $77(61.6)$ & $74(61.2)$ & $70(54.7)$ & 0.457 \\
\hline Shockable.Rhythm & $153(40.9)$ & $63(50.4)$ & $50(41.3)$ & $40(31.2)$ & 0.008 \\
\hline ICU.Death(\%) & $194(51.9)$ & $52(41.6)$ & $62(51.2)$ & $80(62.5)$ & 0.004 \\
\hline Hosp.Death(\%) & $213(57.0)$ & $58(46.4)$ & $66(54.5)$ & $89(69.5)$ & $<0.001$ \\
\hline Unfavourite Outcome in 3 months(\%) & $226(60.4)$ & $61(48.8)$ & $72(59.5)$ & $93(72.7)$ & $<0.001$ \\
\hline Corticoids(\%) & $85(22.7)$ & $22(17.6)$ & $22(18.2)$ & $41(32)$ & 0.008 \\
\hline Chronic.Anticoagulation(\%) & $65(17.4)$ & $28(22.4)$ & $15(12.4)$ & $22(17.2)$ & 0.117 \\
\hline Chron.Heart.Failure(\%) & $78(20.9)$ & $29(23.2)$ & $20(16.5)$ & $29(22.7)$ & 0.361 \\
\hline Hypertension(\%) & $159(42.5)$ & $54(43.2)$ & $53(43.8)$ & $52(40.6)$ & 0.864 \\
\hline Coron.Artery.Dis(\%) & $146(39.0)$ & $54(43.2)$ & $49(40.5)$ & $43(33.6)$ & 0.271 \\
\hline Diabetes(\%) & $91(24.3)$ & $24(19.2)$ & $28(23.1)$ & $39(30.5)$ & 0.105 \\
\hline COPD.Asthma(\%) & $63(16.8)$ & $17(13.6)$ & $25(20.7)$ & $21(16.4)$ & 0.33 \\
\hline Previous.Neurol.Disease(\%) & $54(14.4)$ & $14(11.2)$ & $16(13.2)$ & $24(18.8)$ & 0.209 \\
\hline Chronic.Renal.Failur(\%) & $62(16.6)$ & $17(13.6)$ & $14(11.6)$ & $31(24.2)$ & 0.015 \\
\hline Liver.Cirrhosis(\%) & $17(4.5)$ & $3(2.4)$ & $4(3.3)$ & $10(7.8)$ & 0.086 \\
\hline $\mathrm{IABP}(\%)$ & $24(6.4)$ & $11(8.8)$ & $11(9.1)$ & $2(1.6)$ & 0.022 \\
\hline $\mathrm{ECMO}(\%)$ & $47(12.6)$ & $24(19.2)$ & $8(6.6)$ & $15(11.7)$ & 0.011 \\
\hline Shock(\%) & $200(53.5)$ & $66(52.8)$ & $49(40.5)$ & $85(66.4)$ & $<0.001$ \\
\hline VSP.used(\%) & $283(75.7)$ & $87(69.6)$ & $84(69.4)$ & $112(87.5)$ & $<0.001$ \\
\hline Dobutamine.used(\%) & $201(53.7)$ & $68(54.4)$ & $57(47.1)$ & $76(59.4)$ & 0.15 \\
\hline Mechanical.Ventilation.used(\%) & $369(98.7)$ & $124(99.2)$ & $119(98.3)$ & $126(98.4)$ & 0.867 \\
\hline CRRT.used(\%) & $61(16.3)$ & $16(12.8)$ & $16(13.2)$ & $29(22.7)$ & 0.056 \\
\hline Paracetamol(\%) & $203(54.3)$ & $68(54.4)$ & $69(57)$ & $66(51.6)$ & 0.688 \\
\hline Amidarone(\%) & $187(50.0)$ & $59(47.2)$ & $63(52.1)$ & $65(50.8)$ & 0.73 \\
\hline B.lactams(\%) & $158(42.2)$ & $49(39.2)$ & $56(46.3)$ & $53(41.4)$ & 0.517 \\
\hline FQN(\%) & $7(1.9)$ & $1(0.8)$ & $3(2.5)$ & $3(2.3)$ & 0.652 \\
\hline Azoles(\%) & $8(2.1)$ & $3(2.4)$ & $4(3.3)$ & $1(0.8)$ & 0.333 \\
\hline non.INH(\%) & $374(100.0)$ & $125(100)$ & $121(100)$ & $128(100)$ & 1 \\
\hline non.TMT.SMT(\%) & $374(100.0)$ & $125(100)$ & $121(100)$ & $128(100)$ & 1 \\
\hline Metronidazole(\%) & $2(0.5)$ & $2(1.6)$ & $0(0)$ & $0(0)$ & 0.209 \\
\hline
\end{tabular}




\begin{tabular}{|c|c|c|c|c|c|}
\hline non.Chemotherapy(\%) & $374(100.0)$ & $125(100)$ & $121(100)$ & $128(100)$ & 1 \\
\hline AKI(\%) & $221(59.1)$ & $63(50.4)$ & $66(54.5)$ & $92(71.9)$ & 0.001 \\
\hline Lowest Scv02/Sv02(\%) & $61.0 \pm 8.5$ & $60.6 \pm 9.3$ & $61.6 \pm 8.2$ & $60.8 \pm 8.2$ & 0.635 \\
\hline Min.PLT(/mm3) & $143.6 \pm 89.1$ & $131.6 \pm 74.1$ & $144.3 \pm 77.3$ & $154.8 \pm 109.8$ & 0.119 \\
\hline Lact (mEq l-1) & $6.2 \pm 3.3$ & $6.5 \pm 3.5$ & $5.6 \pm 2.5$ & $6.7 \pm 3.7$ & 0.017 \\
\hline $\mathrm{CRP}(\mathrm{mg} / \mathrm{dl})$ & $62.8 \pm 72.4$ & $50.0 \pm 62.2$ & $57.0 \pm 69.5$ & $80.7 \pm 80.9$ & 0.002 \\
\hline Creat (mg/dl) & $1.5 \pm 1.2$ & $1.4 \pm 0.9$ & $1.4 \pm 1.1$ & $1.7 \pm 1.5$ & 0.026 \\
\hline Scv02/SvO2(\%) & $69.2 \pm 9.1$ & $69.0 \pm 8.7$ & $69.4 \pm 9.1$ & $69.2 \pm 9.6$ & 0.953 \\
\hline GOT (U/L) & $95.0(47.0,192.5)$ & $78.0(39.0,152.0)$ & $101.0(50.0,191.0)$ & $106.0(56.5,235.0)$ & 0.029 \\
\hline GPT (U/L) & $152.3 \pm 374.4$ & $116.4 \pm 218.1$ & $150.1 \pm 251.5$ & $189.3 \pm 550.3$ & 0.302 \\
\hline $\mathrm{LDH}(\mathrm{U} / \mathrm{L})$ & $525.1 \pm 1098.6$ & $387.7 \pm 350.7$ & $503.3 \pm 642.4$ & $679.9 \pm 1729.6$ & 0.103 \\
\hline $\operatorname{ALP}(\mathrm{U} / \mathrm{L})$ & $93.7 \pm 66.7$ & $49.1 \pm 10.9$ & $76.7 \pm 7.9$ & $153.2 \pm 84.2$ & $<0.001$ \\
\hline GGT (U/L) & $101.0 \pm 141.1$ & $59.3 \pm 45.4$ & $91.7 \pm 117.8$ & $150.6 \pm 197.6$ & $<0.001$ \\
\hline Bil.TOT (mg/dl) & $0.9 \pm 1.8$ & $0.7 \pm 1.5$ & $0.8 \pm 0.8$ & $1.3 \pm 2.5$ & 0.017 \\
\hline aPTT(sec) & $43.0 \pm 28.8$ & $47.3 \pm 34.4$ & $38.6 \pm 24.9$ & $42.9 \pm 25.7$ & 0.061 \\
\hline PT(\%) & $63.0 \pm 22.8$ & $60.0 \pm 25.0$ & $67.6 \pm 21.7$ & $61.6 \pm 21.1$ & 0.022 \\
\hline INR & $1.6 \pm 1.2$ & $1.7 \pm 1.2$ & $1.6 \pm 1.4$ & $1.5 \pm 1.1$ & 0.618 \\
\hline $\mathrm{PLT}(/ \mathrm{mm} 3)$ & $208.9 \pm 107.5$ & $188.8 \pm 82.4$ & $216.5 \pm 105.0$ & $221.3 \pm 127.5$ & 0.035 \\
\hline $\operatorname{Prot}(\mathrm{mg} / \mathrm{dl})$ & $7.2 \pm 8.8$ & $6.9 \pm 8.9$ & $6.7 \pm 6.8$ & $8.0 \pm 10.2$ & 0.454 \\
\hline $\mathrm{Glu}(\mathrm{mg} / \mathrm{dl})$ & $234.8 \pm 125.1$ & $232.0 \pm 117.6$ & $252.7 \pm 126.8$ & $220.6 \pm 129.4$ & 0.122 \\
\hline $\mathrm{pH}$ & $7.3 \pm 0.1$ & $7.3 \pm 0.1$ & $7.3 \pm 0.1$ & $7.3 \pm 0.1$ & 0.422 \\
\hline $\mathrm{PO} 2(\mathrm{mmHg})$ & $151.1 \pm 104.2$ & $161.2 \pm 120.7$ & $148.7 \pm 89.0$ & $143.4 \pm 100.1$ & 0.379 \\
\hline $\mathrm{PCO} 2(\mathrm{mmHg})$ & $39.2 \pm 10.9$ & $38.7 \pm 9.6$ & $39.8 \pm 10.1$ & $39.2 \pm 12.6$ & 0.722 \\
\hline $\mathrm{MAP}(\mathrm{mmHg})$ & $90.5 \pm 21.2$ & $89.9 \pm 18.7$ & $92.4 \pm 21.0$ & $89.4 \pm 23.5$ & 0.484 \\
\hline HIGHEST.BIL(mg/dl) & $1.5 \pm 2.5$ & $1.4 \pm 2.5$ & $1.2 \pm 1.4$ & $2.0 \pm 3.1$ & 0.02 \\
\hline HIGHEST.INR & $2.1 \pm 2.3$ & $2.1 \pm 2.3$ & $2.1 \pm 2.7$ & $2.1 \pm 1.9$ & 0.999 \\
\hline
\end{tabular}

$\mathrm{ICU}=$ intensive care unit; $\mathrm{CPR}=$ cardiopulmonary resuscitation; $\mathrm{ROSC}=$ return of spontaneous circulation; $\mathrm{TTM}=$ targeted temperature management;COPD = chronic pulmonary obstructive disease; IABP = intra-aortic balloon counterpulsation; ECMO = extracorporeal membrane oxygenation; CRRT = continuous renal replacement therapy; $\mathrm{AKI}=$ acute liver failure; ScvO2/SvO2 = central venous

oxygen saturation or mixed venous oxygen saturation; AST = aspartate aminotransferase; $A L T=$ alanine aminotransferase; $L D H=$ lactate

dehydrogenase; $\mathrm{ALP}=$ alkaline phosphatase; $\mathrm{GGT}$ = y-glutamine transferase; APTT = activated partial thromboplastin time; $\mathrm{PT}=$ prothrombin time; INR

= international normalized ratio;MAP = mean arterial pressure; CRP = C-reactive protein;

\section{Association between ALP levels and unfavorable neurological outcome in 3 months in the study}

Table 2 shows the ORs and 95\% Cls for risk of UNO in 3 months determined by serum ALP levels. In the extended multivariable logistic regression models,we observed that When included as a continuous variable,the ORs of UNO in 3 months were consistently significant in all six models (ORs range 1.0077-1.0112, $\mathrm{p}<0.05$ for all).ALP was associated with an increased risk of UNO in 3 months (each 10-U/L higher ALP level was associated with a $7.7 \%$ higher hazard (95\% $\mathrm{Cl},(1.0004,1.0151) ; \mathrm{P}=0.0398)$ for UNO in 3 months. The univariate logistic regression analysis revealed that the ORs of UNO in 3 months were $1.5417(0.9306,2.5538)$ and $2.7878(1.6516,4.7057)$ for ALP tertiles $2-3$,respectively $(P$ for trend $=0.00012)$. After adjusting for confounding variables, the the ORs were $1.1029(0.5364,2.2674)$ and $1.7407(0.7676,3.9472)$ for ALP tertiles $2-3$,respectively $(P$ for trend $=0.19709)$. 
Table2

Association between ALP levels and unfavourable neurological outcome in 3 months in the study.

\begin{tabular}{|c|c|c|c|c|c|c|c|c|c|}
\hline & Nonadjusted & & Adjust I & & Adjust II & & Adjust $\otimes$ & & Adjust \ \\
\hline & OR (95\% Cl)凶 & $P$ value & HR (95\% Cl) & $P$ value & HR (95\% Cl) & $P$ value & HR (95\% Cl) & $P$ value & HR (95\% Cl) \\
\hline \multicolumn{10}{|c|}{ Unfavourite Outcome in 3 months } \\
\hline ALP & $\begin{array}{l}1.0088 \\
(1.0039 \sim 1.0138)\end{array}$ & 0.00048 & $\begin{array}{l}1.0086 \\
(1.0037 \sim 1.0135)\end{array}$ & 0.00061 & $\begin{array}{l}1.0094 \\
(1.0041 \sim 1.0147)\end{array}$ & 0.00052 & $\begin{array}{l}1.0095 \\
(1.004 \sim 1.015)\end{array}$ & 0.0007 & $\begin{array}{l}1.0112 \\
(1.0052 \sim 1.017\end{array}$ \\
\hline \multicolumn{10}{|l|}{$\begin{array}{l}\text { ALP } \\
\text { levels }\end{array}$} \\
\hline $\mathrm{T} 1(<64)$ & reference & & reference & & reference & & reference & & reference \\
\hline $\mathrm{T} 2(64,91)$ & $\begin{array}{l}1.5417 \\
(0.9306 \sim 2.5538)\end{array}$ & 0.093 & $\begin{array}{l}1.4658 \\
(0.8752 \sim 2.4549)\end{array}$ & 0.14615 & $\begin{array}{l}1.2913 \\
(0.723 \sim 2.3063)\end{array}$ & 0.38772 & $\begin{array}{l}1.2383 \\
(0.6799 \sim 2.2552)\end{array}$ & 0.48478 & $\begin{array}{l}1.3525 \\
(0.706 \sim 2.5912\end{array}$ \\
\hline T3(>91) & 0.00012 & $<0.001$ & $\begin{array}{l}2.7045 \\
(1.5936 \sim 4.5897)\end{array}$ & 0.00023 & $\begin{array}{l}2.6584 \\
(1.4703 \sim 4.8067)\end{array}$ & 0.00121 & $\begin{array}{l}2.3701 \\
(1.2735 \sim 4.411)\end{array}$ & 0.00647 & $\begin{array}{l}2.9473 \\
(1.4753 \sim 5.887\end{array}$ \\
\hline $\begin{array}{l}\text { PFOR } \\
\text { TREND }\end{array}$ & & 0.00012 & & 0.00023 & & 0.00125 & & 0.00682 & \\
\hline
\end{tabular}

\Logistic regression models were used to estimate odd ratio (OR) and 95\% confidence interval (95\% Cl)Notes: data presented are ORs and 95\% Cls. Adjust I $\mathrm{m}$ model adjusted for adjust I+Weight+Witnessed.Arrest+Bystander.CPR+time.to.ROSC+Epinephrine.total.dose+Out.of.Hospital+Cardiac.Etiology+Shockable.Rh II+Corticoids+Chronic.Anticoagulation+Chron.Heart.Failure+Hypertension+Coron.Artery.Dis+Diabetes+COPD.Asthma+Previous.Neurol.Disease+Chronic.Renal

Adjust $\otimes$ model Adjusted for adjust $\otimes+I C U$. stay+TTM+IABP+ECMO+Shock+VSP.used+Dobutamine.used+Mechanical.Ventilation.used+CRRT.used+MAP.Adjus

Q+Lactate+CRP+Creatinine+ScvO2.SvO2+AST+ALT+LDH+GGT+Total.bilirubin+APTT+PT+INR+Platelets+Proteins+Glucose+pH+PaO2+PaCO2.

In the Figure2, a linear association between UNO in 3 months and serum ALP was identified.Solid lines indicate the estimated risk of UNO in 3 months, and the dotted line represent point-wise 95\% confidence interval adjusted for age+gender+Weight+Witnessed.Arrest+Bystander.CPR+time.to.ROSC+

Epinephrine.total.dose+Out.of.Hospital+Cardiac.Etiology+Shockable.Rhythm+Corticoids+Chronic.Anticoagulation+Chron.Heart.Failure+Hypertension+Coron.Ar

\section{Subgroup analyses of the association between ALP levels on unfavorable neurological outcome in 3 months in the study.}

Shock,Dobutamine.used,Hypertension,Cardiac.Etiology and Shockable.Rhythm are known confounders of ALP and UNO in 3 months association. To see if the association between serum ALP levels and UNO in 3 months is stable in different subgroups, we did stratified analyses and interactive analyses (Figure

3). The association between ALP and UNO in 3 months in the stratified analysis was consistent with that in the multivariable logistic regression analysis. The stratified analysis demonstrated a statistically significant association between ALP levels and UNO in 3 months in non.Dobutamin.used subjects (adjusted $\mathrm{OR}, 1.0324 ; 95 \% \mathrm{Cl}, 1.009-1.0564$ ), non-Hypertension subjects (adjusted OR, 1.018; 95\% Cl, 1.0041-1.032). Data showed that Hypertension played an interactive role in the association between ALP and UNO in 3 months ( $P$ for interaction 0.004).

\section{Discussion}

In this population-based cohort study, ALP was found to be associated with an elevated risk of the UNO in 3 months, independent of age + gender + Weight + Witnessed.Arrest + Bystander.CPR + time.to. ROSC + Epinephrine.total.dose + Out.of.Hospital + Cardiac. Etiology + Shockable.Rhythm + Corticoids + Chronic.Anticoagulation + Chron. Heart.Failure + Hypertension + Coron.Artery.Dis + Diabetes + COPD.Asthma + Previous.Neurol.Disease + Chronic.Renal.Failur + Liver.Cirrhosis + ICU.stay + TTM + IABP + ECMO + Shock + VSP.used + Dobutamine.used + Mechanical.Ventilation.used + CRRT.used + MAP + Lactate + CRP + Creatinine + Scv02.SvO2 + AST + ALT + LDH + GGT + Total.bilirubin + APTT + PT + INR + Platelets + Proteins + Glucose + pH + PaO2 + PaCO2. We revealed a linear relationship between serum ALP levels and the risk of the UNO in 3 months. The relationship was characterized as follows: A $7.7 \%$ higher risk of UNO in 3 months, associated with a $10 \mathrm{U} / \mathrm{L}$ increased in serum ALP. In addition, in the multi-factor analysis (Table 2), we could also find that the relationship between the ALP and the prognostic becomes more obvious as the ALP increases,especially obvious in the T3 group.After adjustment, ALP increased 1 unit, the prognosis risk increased $74.07 \%$ (1.7407 (0.7676,3.9472)), while the corresponding T2 groups increased only $10.29 \%(1.1029(0.5364,2.2674))$.In the stratified analysis, we observed significant associations in patients with non-hypertension, but not in patients with hypertension.Interestingly, we also observed significant associations in the non-Dobutamine.used group, despite no interaction between ALP and the results. We found no interaction in the shock, cardiac.Etiology,shockable.Rhythm groups, indicating that the relationship between ALP and the results was unrelated to the cardiogenic cause, defibrillation rhythm, and shock.

ALP, an enzyme first discovered in the $1920 \mathrm{~s}$, has been evaluated as a potential biomarker for many diseases ${ }^{6}$. The rise in ALP is a common manifestation of ischemic stroke. It has been made clear that ALP is an independent risk factor for the adverse prognosis of ischemic stroke and has a positive correlation ${ }^{13,23,}$

${ }^{24}$.Neurodysfunction of ischemic stroke mainly comes from ischemic damage of nerve cells. The injury to brain cells in patients after CA also mainly stems from ischemic and hypoxia injury during cardiac arrest and reperfusion injury after recovery.It shows that the two have some similarities, and our results suggest that the positive correlation of ALP with bad prognosis also agrees with previous studies. 
The reasons for ALP rise in CA may be the following:First, some basic studies have shown that ALP activity can be expressed in brain endothelial cells and neurons ${ }^{8,9}$.An association between ALP and minor cerebrovascular disease was found by Lee et al ${ }^{10}$. When blood supply disorder occurs in the arterial artery, the corresponding brain tissue develops ischemia, damage or even necrosis, and blood ALP increases. The greater the infarct range, the higher the ALP, the more obvious the corresponding nerve function damage. Brichacek et al. also found that ALP caused adverse outcomes by playing a role in the broken bloodbrain barrier (BBB), neurological inflammation, and vascular dysfunction in stroke patients ${ }^{6}$. Because small areas of cerebral infarction are often associated with a neurologic prognosis, this explains why OR increases in ALP > 91 and ALP $<64$.Second, serum ALP levels are also associated with inflammation or malnutrition. Similar to the C-reactive protein, ALP also reflects the inflammatory state of the body ${ }^{25}$. The reperfusion after prolonged cardiac arrest ischemia leads to increased inflammatory cytokines ${ }^{26}$.Due to the systemic inflammatory response, patients after CA showed a sepsis-like state.In fact, in this study, elevated ALP levels were associated with CRP levels and several nutritional states.However, we found that the elevation of ALP levels and the adverse stroke outcome remained stable after adjusting these indicators. Thus, it is unlikely for the link between ALP and adverse outcomes of stroke to rely solely on malnutrition and inflammation. However, some studies conclude not exactly the same as ours.It was found that ALP was not significantly associated with neurofunctional results from a year later ${ }^{20}$. The reason may be the grouping of ALP was different.Their study was based on the ALP grouping of the ending, while our research was based on grouping by the ALP value.

In the subgroup analysis,Our subgroup analysis revealed that the association between ALP and risk of UNO in 3 months existed between the layers except hypertension. It showed that ALP concentration was positively associated with UNO in 3 months in nonhypertension (OR 1.018(1.0041,1.032)), $P$ for trend 0.01083)However, the associations were not observed in hypertensions. The reasons for the unclear relationship between ALP and the adverse outcome in the hypertension group were unclear.lt has been proposed that there are various complications in patients with hypertension, so TTM is associated with good neurological recovery in patients without hypertension, but not in patients with hypertension ${ }^{27,28}$. Eujene Jung suggests that hypertension does not have an adverse effect on the prognosis of patients with cardiac arrest ${ }^{27}$. This may be associated with hypertension patients with greater awareness of activating emergency medical services (EMS) with ischemic symptoms.And lead to short cardiac arrest to EMS recovery ${ }^{29}$.Another possibility is that the effects of hypertension drugs can be taken into account. There have been studies that calcium channel blockers can be treated in ischemic pretreatment reserved with cardiac arrest and angiotensin converting enzyme inhibitors to reduce recurrent myocardial infarction and ischemia ${ }^{30,31}$.In addition, most patients with hypertension have cerebrovascular sclerosis, which involves too many diseases ${ }^{32}$.In the future, it is necessary to study the factors related to HTN which improve the favorable neurological outcome of CA patients.

This study has several limitations. First, this is a population-based observational study, not an intervention study. There are likely to be significant potential biases that were not considered.Second,because we studied the population of patients who recovered heart breathing after CA, we excluded the early death ( $\mathrm{n}$ $=51$ ) to reduce bias. Also, the dependent variable we studied ALP is one of the liver enzymes, so we excluded the absence of data on liver transaminases, coagulation, and/or total bilirubin $(n=10)$. This leads to a reduced number of studies, but after regression analysis and hierarchical analysis,the results remain robust and reliable. Third, compared to previous studies, we lack variate data like hypertension medication, however, our data have 48 covariates that reduce the bias as possible, and our results all show consistency after adjusting the covariates, which also shows that our results are stable.These limitations should be considered in future studies.

\section{Conclusion}

ALP is an independent risk factor for the UNO in 3 months after CA, especially in non-hypertension. Elevated ALP was significantly associated with increased UNO in 3 months after CA. This is similar to the relationship between ALP and the prognosis of ischemic stroke. However, the prediction significance of ALP for long-term neurological outcome in patients after CA is needed.

\section{Declarations}

\section{Funding}

None

\section{Availability of data and material}

Data can be downloaded from the DRYAD database (http:// www.Datadryad.org).

\section{Competing interests}

The author declares no conflicts of interest in this work.

\section{Consent to publish}

Not applicable/all data used for the present study have been anonymized, and the submission does not include information that may identify individual persons. 


\section{Acknowledgments}

The authors are very grateful to the data providers of the study. They completed the entire study. They are Enrica lesu, Federico Franchi, Federica Zama Cavicchi, Selene Pozzebon, Vito Fontana, Manuel Mendoza, Leda Nobile, Sabino Scolletta, Jean-Louis Vincent, Jacques Creteur, Fabio Silvio Taccone (corresponding author) (the rankings of these researchers were ranked according to reference [14]).

\section{References}

1. Field, J. M. et al. Part 1: executive summary: 2010 American Heart Association Guidelines for Cardiopulmonary Resuscitation and Emergency Cardiovascular Care. Circulation. 2010-11-02 2010;122(18 Suppl 3):S640-S656.

2. Neumar, R. W. et al. Part 8: adult advanced cardiovascular life support: 2010 American Heart Association Guidelines for Cardiopulmonary Resuscitation and Emergency Cardiovascular Care. Circulation. 2010-11-02 2010;122(18 Suppl 3):S729-S767.

3. Nolan, J. P. et al. European Resuscitation Council and European Society of Intensive Care Medicine Guidelines for Post-resuscitation Care 2015: Sect. 5 of the European Resuscitation Council Guidelines for Resuscitation 2015. Resuscitation. 2015-10-01 2015;95:202-222.

4. Stammet, P. et al. Neuron-Specific Enolase as a Predictor of Death or Poor Neurological Outcome After Out-of-Hospital Cardiac Arrest and Targeted Temperature Management at 33 degrees C and 36 degrees C. J Am Coll Cardiol. 2015-05-19 2015;65(19):2104-2114.

5. Bascom, K. E. et al. Derivation and Validation of the CREST Model for Very Early Prediction of Circulatory Etiology Death in Patients Without ST-SegmentElevation Myocardial Infarction After Cardiac Arrest. Circulation. 2018-01-16 2018;137(3):273-282.

6. Brichacek, A. L. \& Brown, C. M. Alkaline phosphatase: a potential biomarker for stroke and implications for treatment. Metab Brain Dis. 2019-02-01 2019;34(1):3-19.

7. Moss, D. W. Alkaline phosphatase isoenzymes. Clin Chem. 1982-10-01 1982;28(10):2007-2016.

8. Ermonval, M. et al. The cellular prion protein interacts with the tissue non-specific alkaline phosphatase in membrane microdomains of bioaminergic neuronal cells. Plos One. 2009-08-04 2009;4(8):e6497.

9. Brun-Heath, I. et al. Differential expression of the bone and the liver tissue non-specific alkaline phosphatase isoforms in brain tissues. Cell Tissue Res. 2011-03-01 2011;343(3):521-536.

10. Lee, H. B. et al. Association between Serum Alkaline Phosphatase Level and Cerebral Small Vessel Disease. Plos One. 2015-01-20 2015;10(11):e143355.

11. Jia, H., Li, H., Liu, Y., Liu, C. \& Xue, M. Elevated serum alkaline phosphatase as a predictor of cognitive impairment in patients with acute ischaemic stroke: A retrospective cohort study. Arch Gerontol Geriatr. 2020-07-01 2020;89:104104.

12. Zhong, C. et al. Serum Alkaline Phosphatase, Phosphate, and In-Hospital Mortality in Acute Ischemic Stroke Patients. J Stroke Cerebrovasc Dis. 2018-0101 2018;27(1):257-266.

13. Zong, L. et al. Alkaline Phosphatase and Outcomes in Patients With Preserved Renal Function: Results From China National Stroke Registry. Stroke. 201805-01 2018;49(5):1176-1182.

14. lesu, E. et al. Acute liver dysfunction after cardiac arrest. Plos One. 2018-01-20 2018;13(11):e206655.

15. Tujjar, O. et al. Acute kidney injury after cardiac arrest. Crit Care. 2015-04-17 2015;19:169.

16. Taccone, F. et al. How to assess prognosis after cardiac arrest and therapeutic hypothermia. Crit Care. 2014-01-14 2014;18(1):202.

17. Vincent, J. L. et al. The SOFA (Sepsis-related Organ Failure Assessment) score to describe organ dysfunction/failure. On behalf of the Working Group on Sepsis-Related Problems of the European Society of Intensive Care Medicine. Intensive Care Med. 1996-07-01 1996;22(7):707-710.

18. Jennett, B. \& Bond, M. Assessment of outcome after severe brain damage. Lancet. 1975-03-01 1975;1(7905):480-484.

19. Kim, J. et al. Serum alkaline phosphatase and phosphate in cerebral atherosclerosis and functional outcomes after cerebral infarction. Stroke. 2013-12-01 2013;44(12):3547-3549.

20. Liu, Y. et al. Increased Serum Alkaline Phosphatase in Patients with Acute Ischemic Stroke. J Stroke Cerebrovasc Dis. 2019-01-01 2019;28(1):21-25.

21. Fruh, A. et al. Gastric regurgitation predicts neurological outcome in out-of-hospital cardiac arrest survivors. Eur J Intern Med. 2021-01-01 2021;83:54-57.

22. Thorgeirsdottir, B. et al. Plasma proenkephalin A 119-159 and dipeptidyl peptidase 3 on admission after cardiac arrest help predict long-term neurological outcome. Resuscitation. 2021-06-01 2021;163:108-115.

23. Ryu, W. S., Lee, S. H., Kim, C. K., Kim, B. J. \& Yoon, B. W. Increased serum alkaline phosphatase as a predictor of long-term mortality after stroke. Neurology. 2010-11-30 2010;75(22):1995-2002.

24. Nezu, T. et al. Predictors of Stroke Outcome Extracted from Multivariate Linear Discriminant Analysis or Neural Network Analysis. J Atheroscler Thromb. 2020-12-09 2020.

25. Mitaki, S., Nagai, A., Oguro, H. \& Yamaguchi, S. C-reactive protein levels are associated with cerebral small vessel-related lesions. Acta Neurol Scand. 201601-01 2016;133(1):68-74.

26. Adrie, C. et al. Successful cardiopulmonary resuscitation after cardiac arrest as a "sepsis-like" syndrome. Circulation. 2002-07-30 2002;106(5):562-568.

27. Jung, E. et al. Effect of hypertension across the age group on survival outcomes in out-of-hospital cardiac arrest. Am J Emerg Med. 2019-04-01 2019;37(4):608-614.

28. Jung, E. et al. Interaction Effects Between Targeted Temperature Management and Hypertension on Survival Outcomes After Out-of-Hospital Cardiac Arrest: A National Observational Study from 2009 to 2016. Ther Hypothermia Temp Manag. 2020-09-01 2020;10(3):141-147. 
29. Lee, M. H. et al. Effect of known history of heart disease on survival outcomes after out-of-hospital cardiac arrests. Emerg Med Australas. 2018-02-01 2018;30(1):67-76.

30. Domanski, M. J. et al. Effect of angiotensin converting enzyme inhibition on sudden cardiac death in patients following acute myocardial infarction. A meta-analysis of randomized clinical trials. J Am Coll Cardiol. 1999-03-01 1999;33(3):598-604.

31. Stecker, E. C. et al. Ischemic heart disease diagnosed before sudden cardiac arrest is independently associated with improved survival. $J$ Am Heart Assoc. 2014-10-06 2014;3(5):e1160.

32. Pires, P. W., Dams, R. C., Matin, N. \& Dorrance, A. M. The effects of hypertension on the cerebral circulation. Am J Physiol Heart Circ Physiol. 2013-06-15 2013;304(12):H1598-H1614.

\section{Figures}

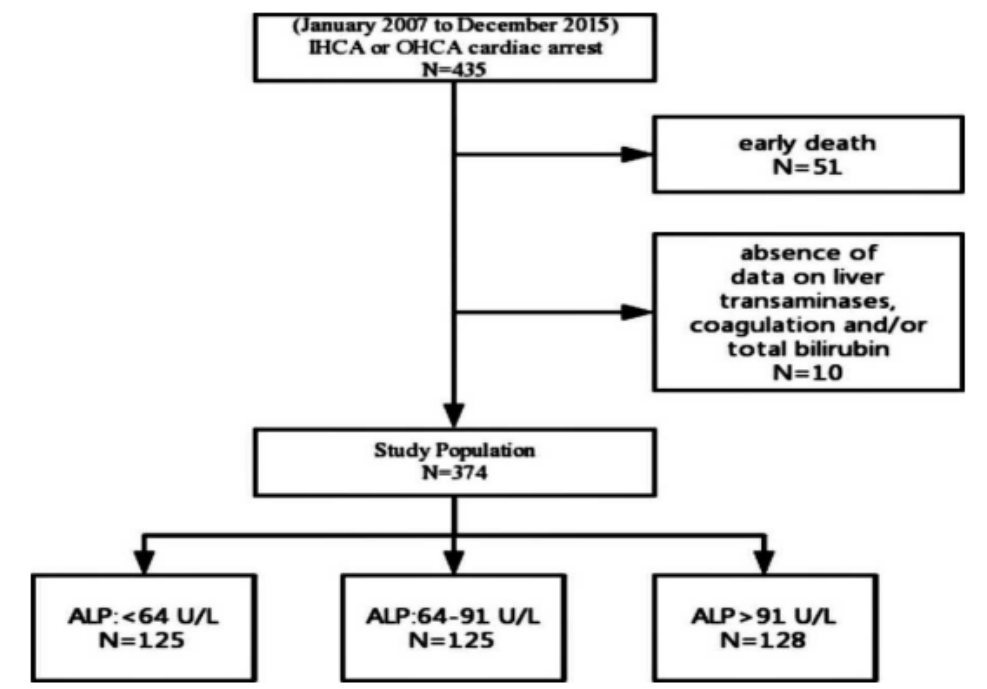

Figure 1:Flow diagram of the screening and enrollment of study participants.Abbreviations: ALP = alkaline phosphatase, $\mathrm{IHCA}=$ in hospital cardiac arrest, OHCA:out of hospital cardiac arrest.

\section{Figure 1}

See image above for figure legend. 


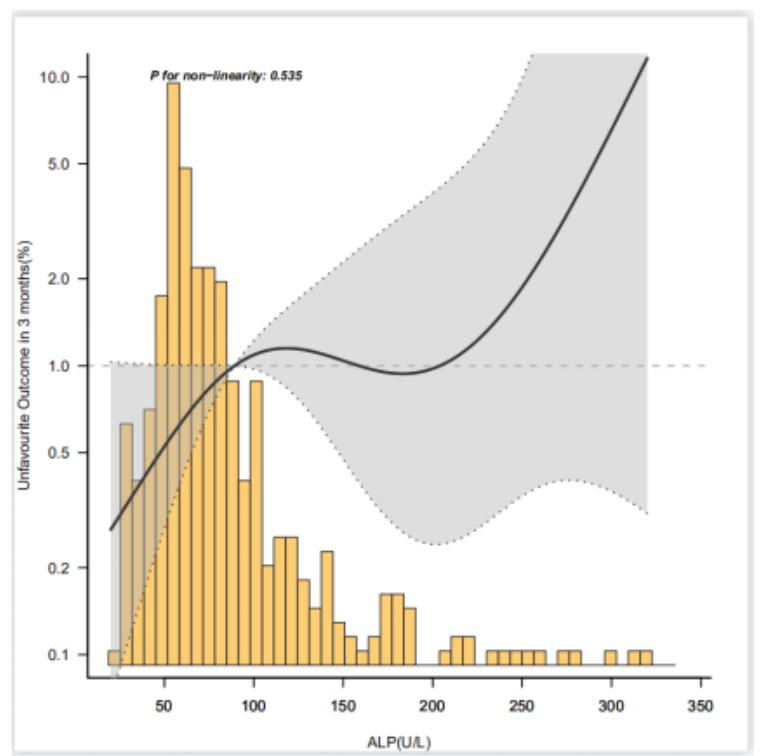

Figure 2fitting a curve for unfavourable neurological outcome in 3 months according to the ALP.Adjusted for age+gender+ Weight+Witmessed.Arrest+Bystander.CPR+time. to ROSC-Epinephrine. total.dose+Out.of.Hospital+Cardiac.Etiology+ Shockable.Rhythm+Corticoids+Chronic.Anticosgulation+Chron. Heart.Failure-Hypertension+Coron.Artery.Dis+Disbetes+CoPD.Asthm a+Previous. Neurol.Disease+Chronic.Renal.Failur-Liver. Cirmosis+ICU stay+TTM+IABP+ECMO+Shock+VSP.used+Dobutamine. usedMechanical. Ventilation. used+CRRT. used +MAP+Lactate+CRP+Creatinine+ScvO2.SvO2+AST+ALT+LDH+GGT+Total. bilinubin-APT $\mathrm{T}+\mathrm{PT}+\mathrm{NNR}+\mathrm{Platalets}+\mathrm{Proteins}+\mathrm{Glucose}+\mathrm{pH}+\mathrm{PaO} 2+\mathrm{PaCO} 2$.

Figure 2

See image above for figure legend.

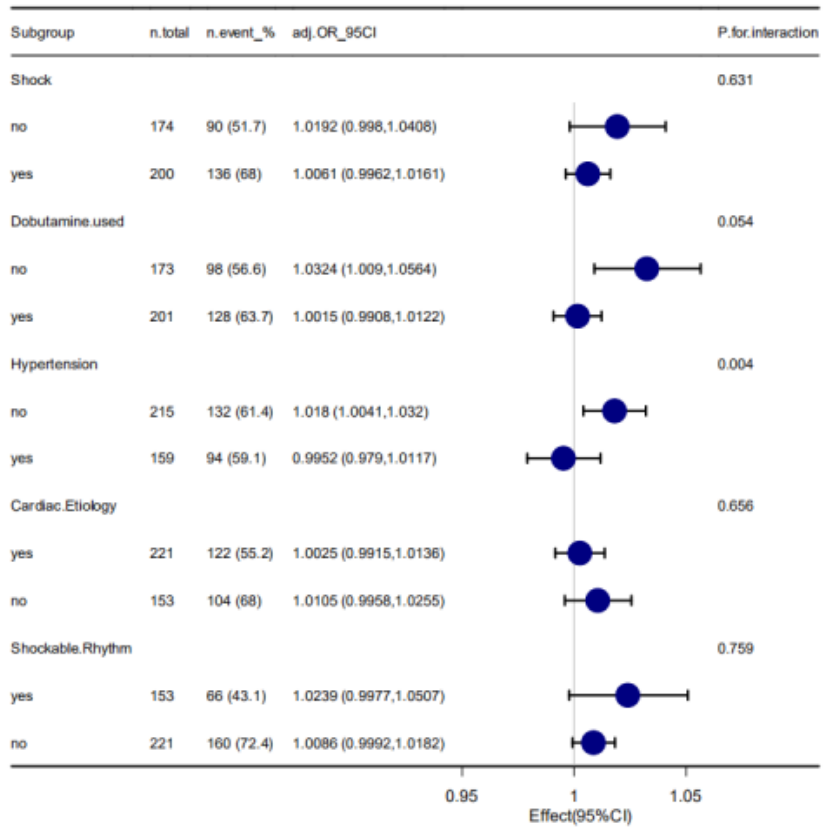

Figure 3. Odds ratio $(95 \% \mathrm{Cl})$ for the unfavourable neurological outcome in 3 months in the study.

\section{Figure 3}

See image above for figure legend. 


\section{Supplementary Files}

This is a list of supplementary files associated with this preprint. Click to download.

- Table3.docx 